\title{
Influence of irradiance and temperature on the iron content of the marine diatom Thalassiosira weissflogii (Bacillariophyceae)
}

\author{
R. F. Strzepek ${ }^{*}$, N. M. Price \\ Department of Biology, McGill University, 1205 Ave. Dr. Penfield, Montreal, Quebec H3A 1B1, Canada
}

\begin{abstract}
The marine diatom Thalassiosira weissflogii (Grun.) Fryxell et Hasle was grown over a wide range of irradiances and temperatures in both $\mathrm{NO}_{3}{ }^{-}$and $\mathrm{NH}_{4}{ }^{+}$-amended seawater containing high Fe. Maximum growth rates were not affected by $\mathrm{N}$ source, but the initial slope of the growth irradiance curve was significantly greater for the $\mathrm{NH}_{4}{ }^{+}$-grown cells. Light-limited phytoplankton con-

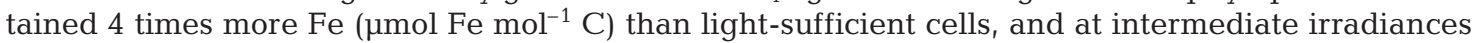
had the highest chlorophyll a concentrations $\left(\mathrm{g} \mathrm{C} \mathrm{g}^{-1} \mathrm{chl} a\right)$. Iron quotas increased as irradiance was reduced because growth rates declined by 20 -fold, whereas steady-state Fe transport rates declined by only a factor of 6 . Accumulation of Fe was apparently regulated by a light-induced physiological requirement. Similar reductions in growth rate with declining temperature elicited only minor changes in Fe quota, and the increase in Fe quota, was observed only at the 2 lowest temperatures. At irradiance levels between 37 and $150 \mu \mathrm{mol}$ photons $\mathrm{m}^{-2} \mathrm{~s}^{-1}$, the relative increase in Fe quota exactly matched the relative increase predicted from the change in abundance of PSI and PSII reported to occur during photoacclimation of T. weissflogii. Subsequent increases in Fe quota at low light may represent additional changes to the photosynthetic apparatus or accumulation of Fe in a non-metabolic pool. The results demonstrate that the Fe content of phytoplankton is influenced by ambient light and imply that the extent of photoacclimation of natural populations may be constrained by the availability of Fe.
\end{abstract}

KEY WORDS: Iron quota · Nitrogen · Photoacclimation · Temperature $\cdot$ Thalassiosira weissflogii

\section{INTRODUCTION}

Reduced chlorophyll a content and photosynthetic activity are among the most noticeable effects of iron (Fe) deficiency in phytoplankton, reflecting the requirements for Fe in porphyrin biosynthesis and in the redox catalysts of the photosynthetic electron-transport chain. The photosynthetic cytochromes and Fe-S proteins are quantitatively the most significant part of the catalytic Fe pool in photoautotrophs (Raven 1990).

*Present address: Department of Botany, University of British Columbia, 6270 University Boulevard, Vancouver, British Columbia V6T 1Z4, Canada.

E-mail: strzepek@interchange.ubc.ca
Their abundances and activities are altered by Fe limitation, leading to reduced photosynthetic rates and efficiency (Greene et al. 1991, 1992, Geider et al. 1993). Smaller amounts of $\mathrm{Fe}$ are associated with nitrate assimilation and oxygen-cycling enzymes, and the reactions they catalyze are similarly under the influence of the cellular Fe nutritional state. In low Fe environments of the open sea, activity of the Fe-dependent reactions of photosynthesis and nitrate assimilation are reduced (Price et al. 1991, 1994, Kolber et al. 1994, Behrenfeld et al. 1996). Natural rates of Fe supply are too slow to sustain maximum metabolic rates.

Iron requirements of photoautotrophs are modulated by environmental and chemical factors that influence the need for certain Fe-dependent biochemical reac- 
tions. Nitrate, for example, increases cellular Fe quota compared to ammonium, as it induces synthesis of Fecontaining assimilatory enzymes and requires more reductant to be incorporated into amino acids. The additional Fe required to process nitrate is substantial in diatoms, amounting to about $30 \mu \mathrm{mol} \mathrm{Fe} \mathrm{l}^{-1}$ cell volume (Maldonado \& Price 1996). Light may also increase cellular Fe requirements for phytoplankton because it affects the photosynthetic apparatus. Phytoplankton acclimate to low photon-flux densities by changing pigmentation, and abundance and stoichiometry of Fe-rich electron-transport components (Falkowski et al. 1981, Dubinsky et al. 1986, Friedman \& Alberte 1986, Neile \& Melis 1986, Falkowski \& La Roche 1991). Photoacclimation is widely observed in oceanic phytoplankton, and is important in maintaining optimal photosynthetic rates. The costs of such an acclimation, however, are predicted to be high when light levels are near the compensation irradiance. Raven's (1990) calculation predicts a $\sim 50$-fold increase in Fe demand for photoautotrophic growth at low light compared to full sunlight. Nitrogen source may modify this effect because of differences in the need for reductant in nitrate- and ammonium-grown cells. Light limitation, which impedes production of cellular reductant, should thus be more deleterious when nitrate is the $\mathrm{N}$ source (Thompson et al. 1989). Iron requirements for nitrate use might therefore be more difficult to satisfy when light decreases.

The effect of light on phytoplankton Fe nutrition is further complicated by photochemical reactions that influence Fe chemistry in seawater. Photoreductive dissolution of Fe colloids and Fe(III)-EDTA complexes increases inorganic Fe(II) and, indirectly through subsequent reoxidation, Fe(III) concentrations, promoting phytoplankton growth under Fe-limiting conditions (Sunda \& Huntsman 1995, Wells et al. 1995). Even at relatively low irradiances (e.g. $150 \mu \mathrm{mol}$ photons $\mathrm{m}^{-2}$ $\mathrm{s}^{-1}$ ) this process maintains considerably higher steadystate ferric ion concentrations than would exist by thermal dissolution alone (Wells et al. 1995). As the efficacy of the reaction is wavelength- and intensity-dependent, low-light phytoplankton could be even more Festressed if availability decreases and requirement increases simultaneously. Changes in Fe availability mediated by light are well documented (Wells et al. 1995). Likewise, a photo-induced increase in phytoplankton Fe requirements has recently been established (Kudo \& Harrison 1997, Sunda \& Huntsman 1997).

Temperature, like irradiance, is extremely variable in the ocean, but over different temporal and spatial scales. It influences the balance between energy production and expenditure and hence might modify the functional organization of the photosynthetic appara- tus (Davison 1991). Chlorosis is commonly observed at low temperatures (Geider 1987, Maxwell et al. 1994, $1995 a, b)$. Less frequently measured is photosystem abundance, which is reported to decrease or to remain constant as temperature decreases (Davison 1991). Since the activities and abundance of the reaction centers are Fe-dependent, but carbon fixation enzymes are not, low temperature may have little or no effect on cellular Fe requirements.

We tested some of these predictions by measuring $\mathrm{Fe}$ quotas of the marine diatom Thalassiosira weissflogii grown over a broad range of irradiances and temperatures in Fe-rich seawater. The results show that the Fe content of this photoautotroph increases remarkably under low light and is largely invariant with temperature. Part of the light-induced increase in Fe quota could be accounted for by changes in the biologically active Fe-containing photosynthetic components, suggesting that photoacclimation imparts a significant Fe requirement to this marine diatom.

\section{MATERIALS AND METHODS}

The marine diatom Thalassiosira weissflogii (Clone Actin) was obtained from the Center for Culture of Marine Phytoplankton, Bigelow Laboratory for Ocean Sciences, West Boothbay Harbor, Maine, USA (CCMP 1336). Stock cultures were maintained in polycarbonate tubes at $150 \mu \mathrm{mol}$ photons $\mathrm{m}^{-2} \mathrm{~s}^{-1}$. Although sterile technique was used throughout the experiments, cultures were not axenic. However, measurements showed that bacterial biovolumes in our cultures were negligible compared to those of phytoplankton (Coulter Counter $^{\circledR}$ model ZM). Moreover, most bacteria were removed when the samples were collected on $3 \mu \mathrm{m}$ filters for analysis.

Culture media. Phytoplankton were grown in the artificial seawater medium Aquil (Morel et al. 1979, Price et al. 1988/1989) containing $10 \mu \mathrm{M}$ phosphate $\left(\mathrm{PO}_{4}{ }^{3-}\right), 100 \mu \mathrm{M}$ silicate $\left(\mathrm{SiO}_{3}{ }^{2-}\right)$, and either $100 \mu \mathrm{M}$ nitrate $\left(\mathrm{NO}_{3}^{-}\right)$or $100 \mu \mathrm{M}$ ammonium $\left(\mathrm{NH}_{4}^{+}\right)$. Extraneous trace-metal contaminants were removed from synthetic ocean water (SOW) and nutrient-enrichment stock solutions using Chelex 100 ion-exchange resin (Bio-Rad Laboratories, Richmond, CA, USA) according to the procedure of Price et al. (1988/1989). Media preparation and storage were as described in Maldonado \& Price (1996). Media were enriched with filtersterilized (0.2 $\mu \mathrm{m}$ Acrodisc) EDTA-trace metal and f/2 vitamin solutions (Price et al. 1988/1989). Pre-mixed Fe-EDTA (1:1) was filter-sterilized and added separately to achieve a final concentration of $8.4 \mu \mathrm{M}$, corresponding to an estimated inorganic Fe (Fe') concentration of 41 nM (Maldonado \& Price 1996). Free trace- 
metal ion concentrations, in the presence of $100 \mu \mathrm{M}$ EDTA as the chelating agent, were as follows (-log free metal ion concentration $=$ pMetal): pFe 18.18, pCu 13.79, pMn 8.27, pZn 10.88, and pCo 10.88. These concentrations were calculated using the chemical equilibrium computer program MINEQL (Westall et al. 1976) with the thermodynamic constants reported in Ringbom (1963). Selenite and molybdate were added at $10^{-8}$ and $10^{-7} \mathrm{M}$, respectively.

Growth rate determination. Semi-continuous batch cultures were maintained under continuous illumination in acid-washed, microwave-sterilized $28 \mathrm{ml}$ polycarbonate tubes. Initial inocula for all cultures came from stock cultures that had previously been acclimated to growth on either $\mathrm{NO}_{3}{ }^{-}$or $\mathrm{NH}_{4}{ }^{+}$. Dilutions exceeded 1:100, assuring at least 8 generations per transfer. In vivo chlorophyll a fluorescence of cultures was measured using a Turner Designs model 10-AU fluorometer (Brand et al. 1981), and specific growth rates were determined from least-squares regressions of ln in vivo fluorescence versus time during the exponential phase of growth. Acclimation was assumed complete when the coefficient of variation of growth rates between successive transfers did not vary by more than $10 \%$. The acclimation period for slow-growing cultures (low temperature and low light) exceeded 3 mo.

Experimental design: light experiment. Triplicate cultures were grown in an incubator maintained at $20 \pm 1^{\circ} \mathrm{C}$. Continuous light was provided from below by 4 fluorescent lights (Philips cool white) and attenuated with neutral-density screening. Varying the number of layers of screening established the following photon-flux densities: $6,10,14,20,37,66$, and $108 \mu \mathrm{mol}$ photons $\mathrm{m}^{-2} \mathrm{~s}^{-1}$ (Biospherical Instruments model QSL-100 $4 \pi$ quantum meter, San Diego). A $150 \mu \mathrm{mol}$ photons $\mathrm{m}^{-2} \mathrm{~s}^{-1}$-grown stock culture was used to inoculate all cultures. A second independent trial was conducted 6 mo later following the same experimental protocol, but with an additional irradiance treatment of $150 \mu \mathrm{mol}$ photons $\mathrm{m}^{-2} \mathrm{~s}^{-1}$. Only growth rates were determined for 2 additional treatments of 200 and $600 \mu \mathrm{mol}$ photons $\mathrm{m}^{-2} \mathrm{~s}^{-1}$. An exponential equation modified from Platt \& Gallegos (1980) was used to fit the specific growth rate $(\mu)$ versus irradiance $(I)$ data using non-linear regression.

Experimental design: temperature experiment. Cultures tubes were housed within an aluminum block to which a temperature gradient had been applied with refrigerating and heating units. Quadruplicate $\mathrm{NO}_{3}{ }^{-}-$ and $\mathrm{NH}_{4}{ }^{+}$-preacclimated cultures were inoculated and placed at each of the following temperatures: 10, 13, 16, $18,21,24,27$ and $29^{\circ} \mathrm{C}$. Temperatures below $10^{\circ} \mathrm{C}$ supported cell division, but the rate was too slow for experimentation. Cultures were grown under continuous, saturating irradiance $\left(135 \pm 20 \mu \mathrm{mol}\right.$ photons $\left.\mathrm{m}^{-2} \mathrm{~s}^{-1}\right)$ provided by a bank of fluorescent bulbs (Vita-Lite; Duro-Test Corporation, Fairfield, NJ, USA) placed below the cultures. The fluorescent bulbs used in the irradiance and temperature experiments were spectrally similar (4100 and $5500 \mathrm{~K}$, respectively).

Experimental measurements. Cultures were harvested to determine cellular concentrations of $\mathrm{Fe}, \mathrm{C}, \mathrm{N}$ and chlorophyll a. Cellular Fe was measured using the radiotracer ${ }^{55} \mathrm{FeCl}_{3}$ (specific activity 25 to $40 \mathrm{mCi} \mathrm{mg}^{-1}$, DuPont, Canada). Inocula of acclimated cultures were transferred in triplicate (light experiment) or quadruplicate (temperature experiment) to identical media in which $10 \%$ of the total Fe was added as ${ }^{55} \mathrm{Fe}$. Phytoplankton were allowed to complete at least 8 cell divisions before harvesting to ensure uniform labeling of intracellular Fe. Cells in mid-exponential phase were collected by gravity-filtration (flow rates $<5 \mathrm{ml} \mathrm{min}^{-1}$ ) by filtering $20 \mathrm{ml}$ culture aliquots onto $3.0 \mu \mathrm{m}$ polycarbonate membrane filters (Poretics). Samples were exposed to Ti (III) EDTA-citrate-reducing solution for $10 \mathrm{~min}$ to dissolve Fe hydroxides and remove ferric ions bound to the outer membrane surface (Hudson \& Morel 1989). The ${ }^{55} \mathrm{Fe}$ activity was measured by liquid scintillation counting on a LKB Wallace 1215 Rackbeta II scintillation counter following the addition of fluor (EcoLite, ICN, Montréal). Samples were corrected for filter absorption using ${ }^{55} \mathrm{Fe}$-radiotracer medium without cells. Total cellular Fe was calculated using the specific activity of ${ }^{55} \mathrm{Fe}$ in the medium $\left(\mathrm{dpm} \mathrm{mol}{ }^{-1}\right)$ and the particulate ${ }^{55} \mathrm{Fe}(\mathrm{dpm})$, correcting for quenching and decay. A culture subsample was preserved in $0.1 \%$ formalin (Booth 1987) and counted by light microscopy using a Palmer-Maloney chamber. Carbon-specific steady-state Fe uptake rates $\left(\rho^{\mathrm{ss}}\right)$ were calculated from growth and Fe quota data, viz.: $\rho^{\mathrm{ss}}=$ $\mu Q_{\mathrm{Fe}}$, where $\mu=$ growth rate $\left(\mathrm{d}^{-1}\right)$ and $Q_{\mathrm{Fe}}=$ amol $\mathrm{Fe}$ $\mathrm{mol}^{-1} \mathrm{C}$ ).

Carbon and $\mathrm{N}$ quotas were determined in successive transfers using non-radioactive Fe medium. During exponential phase, $20 \mathrm{ml}$ samples were filtered (<100 mm Hg) onto precombusted $25 \mathrm{~mm}$ Whatman $\mathrm{GF} / \mathrm{C}$ filters $\left(450^{\circ} \mathrm{C}\right.$ for $\left.4 \mathrm{~h}\right)$. Filters were analyzed for elemental $\mathrm{C}$ and $\mathrm{N}$ with a Carlo Erba 1106 elemental analyzer. A subsample of each culture was preserved in $0.1 \%$ formalin for measurements of cell volume and density, using a Coulter Counter ${ }^{\circledR}$ previously calibrated with $5 \mu \mathrm{m}$ polystyrene beads. In the second trial of the light experiment, $\mathrm{C}$ quotas were estimated from C:cell volume $\left(\mathrm{mol} \mathrm{C} \mathrm{\mu m}^{-3}\right.$ ) calculated from Trial 1. Carbon quotas for the $150 \mu \mathrm{mol}$ photons $\mathrm{m}^{-2} \mathrm{~s}^{-1}$ treatment of Trial 2 were estimated from C:cell volume calculated for the $108 \mu \mathrm{mol} \mathrm{m} \mathrm{m}^{-2} \mathrm{~s}^{-1}$ treatment of Trial 1.

Chlorophyll a concentrations were determined by in vitro fluorometry (Parsons et al. 1984) on a Turner 
Designs model 10-AU fluorometer calibrated with spectrophotometrically-measured chl a standards (Sigma). Samples were extracted in $90 \%$ acetone in the dark for $24 \mathrm{~h}$ at $4^{\circ} \mathrm{C}$ prior to analysis.

\section{RESULTS}

\section{Light experiment}

Specific growth rates of Thalassiosira weissflogii were substantially reduced at decreased irradiances (Fig. 1). Results of the 2 independent trials were not
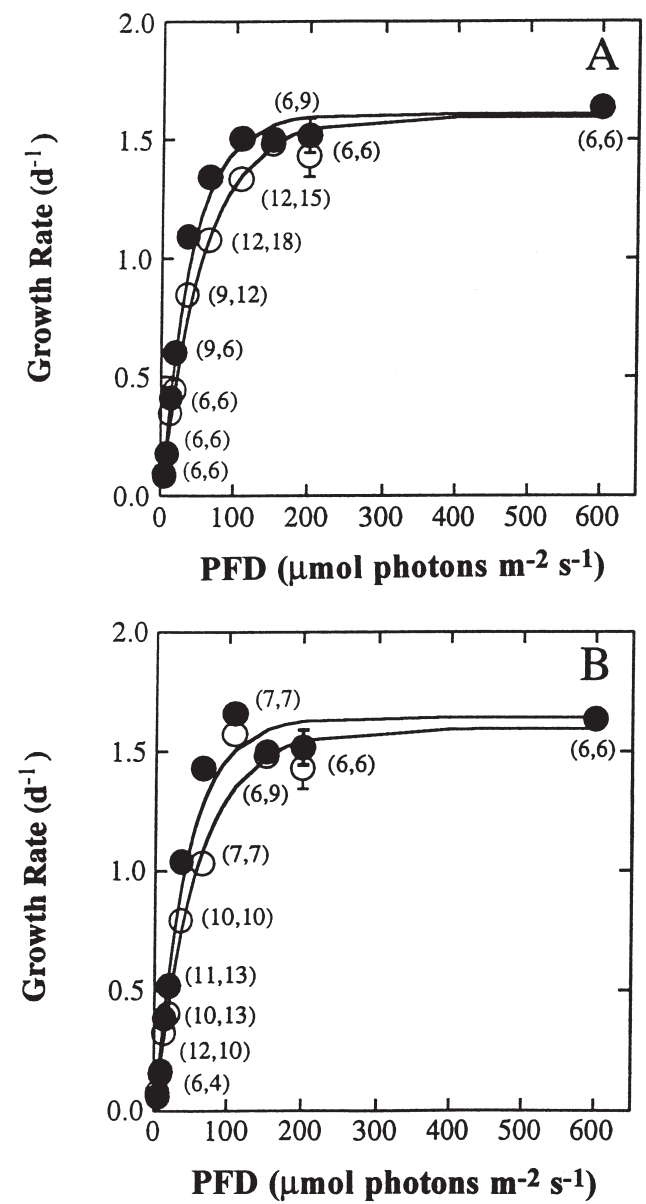

Fig. 1. Thalassiosira weissflogii. Effect of irradiance on specific growth rates $\left(\mathrm{d}^{-1}\right)$ of diatoms grown on Fe-sufficient (pFe 18) Aquil medium amended with either $100 \mu \mathrm{M} \mathrm{NO}_{3}{ }^{-}(\mathrm{O})$ or $\mathrm{NH}_{4}{ }^{+}(\boldsymbol{)})$ at $20^{\circ} \mathrm{C}$. Error bars: $\mathrm{SE}$ around mean (smaller than symbol when not visible); numbers in parentheses: numbers of replicate measurements for $\mathrm{NO}_{3}^{-}$- and $\mathrm{NH}_{4}{ }^{+}$-grown cultures. (A) Trial 1; (B) Trial 2. Following equation modified from Platt \& Gallegos (1980) was fit to data: $\mu=\mu_{\max }\left(1-\mathrm{e}^{-\mathrm{a}}\right)$, where $\mu=$ specific growth rate $\left(\mathrm{d}^{-1}\right) ; a=\alpha_{\mu} I / \mu_{\text {max. }} i \mu_{\max .}=\max$. specific growth rate; $\alpha_{\mu}=$ initial slope of growth curve; $I=$ photon flux density (PFD) significantly different (ANOVA, p > 0.05) and were well described by the exponential equation of Platt \& Gallegos (1980) $\left(\mathrm{r}^{2}>0.97\right.$ for $\mathrm{NO}_{3}{ }^{-}$- and $\mathrm{NH}_{4}{ }^{+}$-grown cultures, both trials). In $\mathrm{NO}_{3}{ }^{-}$-amended media, growth rates ranged 20 -fold from $0.08 \mathrm{~d}^{-1}$ at $6 \mu \mathrm{mol}$ photons $\mathrm{m}^{-2} \mathrm{~s}^{-1}$, to $1.60 \mathrm{~d}^{-1}$ at higher irradiances; $\mathrm{NH}_{4}{ }^{+}-$ amended cultures ranged 24 -fold $\left(0.07\right.$ to $\left.1.65 \mathrm{~d}^{-1}\right)$. Nitrogen substrate did not affect the maximum specific growth rate $\left(\mu_{\text {max. }}\right)$ significantly in either trial (ANOVA, $\mathrm{p}>0.05)$. However, the initial slope of the growth versus irradiance curve $\left(\alpha_{\mu}\right)$ was significantly greater in both trials for $\mathrm{NH}_{4}{ }^{+}$-grown cells (Student's $t$-test, $\mathrm{p}<$ 0.05). Specific growth rates were thus significantly higher for $\mathrm{NH}_{4}{ }^{+}$-grown cells between 37 and $66 \mu \mathrm{mol}$ photons $\mathrm{m}^{-2} \mathrm{~s}^{-1}$ (ANOVA, $\mathrm{p}<0.05$; Tukey multiple means comparison test, $\mathrm{p}<0.05)$. There was no effect of $\mathrm{N}$ substrate on growth rate above and below this irradiance range. Light saturation occurred at ca 83
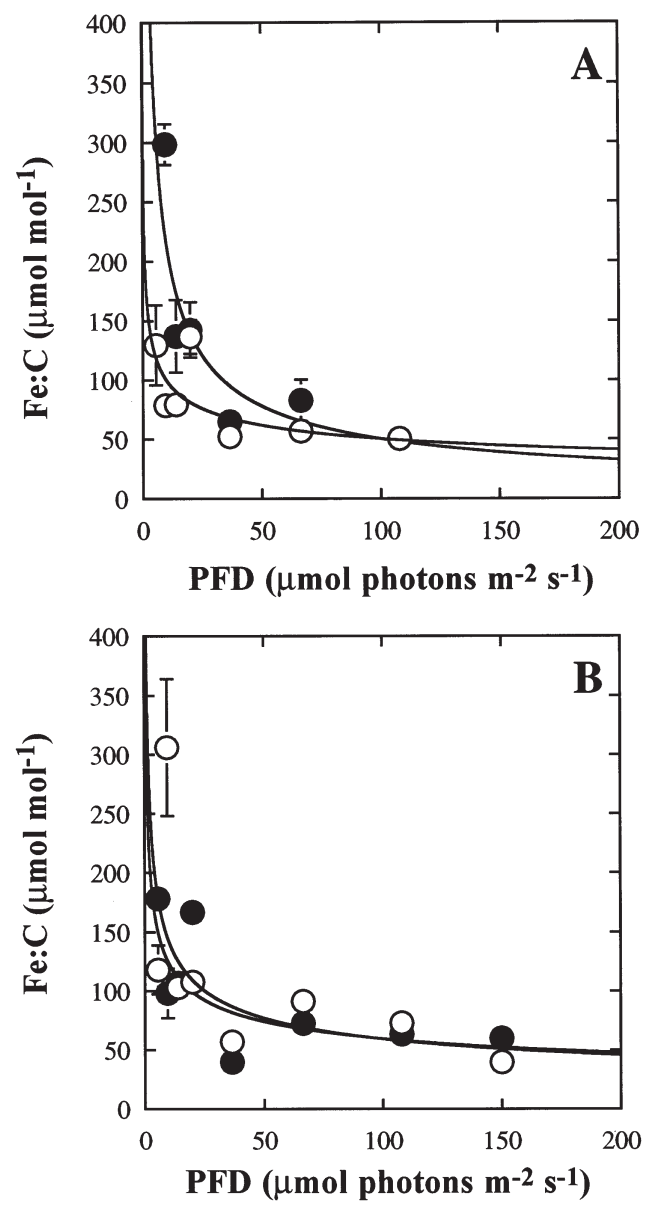

Fig. 2. Thalassiosira weissflogii. Mean Fe:C ratios ( $\mu$ mol Fe $\mathrm{mol}^{-1} \mathrm{C}$ ) of diatoms grown on $100 \mu \mathrm{M} \mathrm{NO}_{3}{ }^{-}(\mathrm{O})$ or $\mathrm{NH}_{4}{ }^{+}(\mathbf{O})$ over range of irradiances at $20^{\circ} \mathrm{C}$. Error bars: SE around mean (smaller than symbol when not visible) $(\mathrm{n}=3)$. A power curve was fit to the data. (A) Trial 1; (B) Trial 2 
and $116 \mu \mathrm{mol}$ photons $\mathrm{m}^{-2} \mathrm{~s}^{-1}$ for $\mathrm{NH}_{4}{ }^{+}$- and $\mathrm{NO}_{3}{ }^{-}$grown cultures, respectively. Cells did not grow below $6 \mu \mathrm{mol}$ photons $\mathrm{m}^{-2} \mathrm{~s}^{-1}$ despite repeated transfers from the next highest irradiance.

Irradiance significantly affected the $\mathrm{Fe}: \mathrm{C}$ ratio $(\mu \mathrm{mol}$ Fe $\mathrm{mol}^{-1} \mathrm{C}$ ) of Thalassiosira weissflogii (Fig. 2). The Fe:C ratio of low-light cultures increased substantially (by up to 4 -fold) relative to high-light cultures (2-way ANOVA, $\mathrm{p}<0.05$; Tukey test, $\mathrm{p}<0.05$ ), in both $\mathrm{NO}_{3}{ }^{-}-$ and $\mathrm{NH}_{4}{ }^{+}$-amended media from both trials. Iron quotas were not affected by $\mathrm{N}$ source (2-way ANOVA, $\mathrm{p}>$ 0.05 ; Tukey test, $\mathrm{p}>0.05$ ).

Intracellular $\mathrm{C}$ varied from 5.5 to $12.3 \mathrm{pmol} \mathrm{cell}^{-1}$ (Table 1); however, most values (11 of 14) were within 9 to $11 \mathrm{pmol} \mathrm{cell}^{-1}$. Intracellular $\mathrm{N}$ varied from 0.9 to $1.3 \mathrm{pmol} \mathrm{cell}{ }^{-1}$. No effect of $\mathrm{N}$ substrate was observed (2-way ANOVAs, p > 0.05; Tukey tests, $p>0.05)$. Cell volumes were invariant over the irradiance range and did not differ significantly (2-way ANOVAs, $\mathrm{p}>0.05$ ) due to $\mathrm{N}$ substrate or between trials (mean \pm SE: $\mathrm{NO}_{3}{ }^{-}=1284 \pm 54 \mu^{3}[\mathrm{n}=14]_{i} \mathrm{NH}_{4}{ }^{+}=$ $1407 \pm 71 \mu^{3}$ [n = 14]). As $\mathrm{C}$ quotas and cell volume were unaffected by light, increases in intracellular Fe quotas at diminished irradiances were due to changes in Fe content alone (Table 1).

Thalassiosira weissflogii responded to decreased irradiance by increasing chl a content (Table 1 ). Maximal chl a content was observed between 20 and 37 $\mu \mathrm{mol}$ photons $\mathrm{m}^{-2} \mathrm{~s}^{-1}$. Carbon:chl a ratios $\left(\mathrm{g} \mathrm{C} \mathrm{g}^{-1} \mathrm{chl}\right.$ a) decreased linearly from 125 to $20 \mu \mathrm{mol}$ photons $\mathrm{m}^{-2} \mathrm{~s}^{-1}$

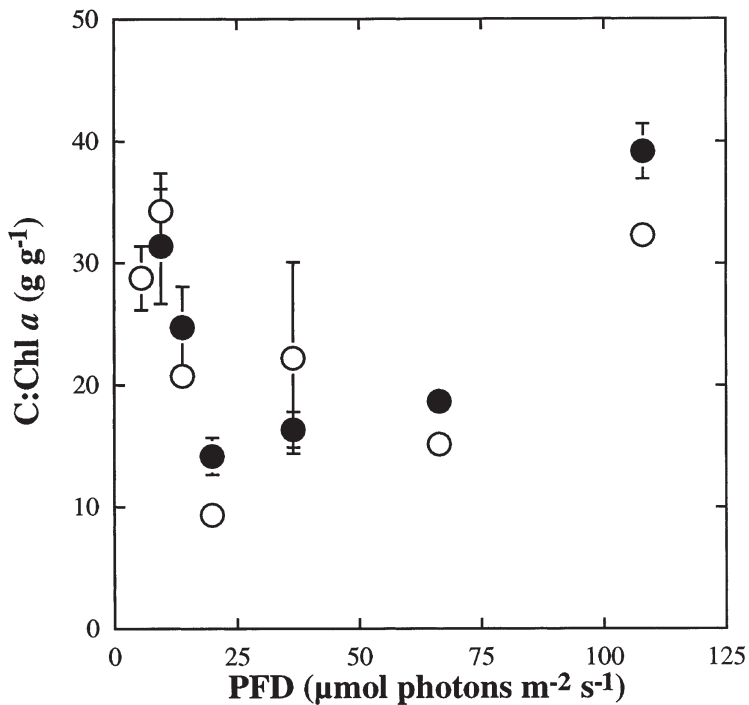

Fig. 3. Thalassiosira weissflogii. Mean C:chlorophyll a ratios $\left(\mathrm{g} \mathrm{g}^{-1}\right.$ ) of diatoms grown on $100 \mu \mathrm{M} \mathrm{NO}_{3}{ }^{-}(\mathrm{O})$ or $\mathrm{NH}_{4}{ }^{+}($) over range of irradiances at $20^{\circ} \mathrm{C}$. Data are from Trial 1. Error bars: SE around mean (smaller than the symbol when not visible) $(n=3)$

as the result of relatively invariant $\mathrm{C}$ and increased chl a (Fig. 3). Below $20 \mu \mathrm{mol}$ photons $\mathrm{m}^{-2} \mathrm{~s}^{-1}$, pigment bleaching occurred. Consequently, at these low irradiances C:chl a reached values similar to high-light cultures. Cellular chl $a$ and C:chl $a$ were not significantly affected by $\mathrm{N}$ substrate (ANOVA, $\mathrm{p}>0.05$ ).

Table 1. Thalassiosira weissflogii. Physiological and biochemical composition parameters of diatoms grown on either $100 \mu \mathrm{M}$ $\mathrm{NO}_{3}{ }^{-}$or $\mathrm{NH}_{4}{ }^{+}$over range of irradiances. T2: results from Trial 2; nm: no measurements taken; values in parentheses: $\mathrm{SEs}(\mathrm{n}=3) ;$ PFD: photon-flux density

\begin{tabular}{|c|c|c|c|c|c|c|c|c|}
\hline $\begin{array}{c}\text { PFD } \\
(\mu \mathrm{mol} \\
\text { photons } \\
\left.\mathrm{m}^{-2} \mathrm{~s}^{-1}\right)\end{array}$ & $\begin{array}{c}\text { Fe } \\
\left(\text { amol cell }^{-1}\right)\end{array}$ & $\begin{array}{c}\text { Fe (T2) } \\
\left(\text { amol cell }^{-1}\right)\end{array}$ & $\begin{array}{c}\mathrm{C} \\
\left(\mathrm{pmol} \mathrm{cell}^{-1}\right)\end{array}$ & $\begin{array}{c}\mathrm{N} \\
\left(\mathrm{pmol} \mathrm{cell}^{-1}\right)\end{array}$ & $\begin{array}{c}\mathrm{C}: \mathrm{N} \\
\left(\mathrm{mol} \mathrm{mol}^{-1}\right)\end{array}$ & $\begin{array}{c}\text { Chl a } \\
\left(\text { pg cell }^{-1}\right)\end{array}$ & $\begin{array}{l}\text { Fe uptake } \\
\text { rate } \\
(\mu \mathrm{mol} \text { Fe n }\end{array}$ & $\begin{array}{r}\text { Fe uptake } \\
\text { rate (T2) } \\
\left.\text { nol }^{-1} \mathrm{C} \mathrm{d}^{-1}\right)\end{array}$ \\
\hline \multicolumn{9}{|c|}{ Nitrate $\left(\mathrm{NO}_{3}{ }^{-}\right)$} \\
\hline 150 & $\mathrm{~nm}$ & $520(26)$ & $\mathrm{nm}$ & $\mathrm{nm}$ & $\mathrm{nm}$ & $\mathrm{nm}$ & $\mathrm{nm}$ & $58.9(3.0)$ \\
\hline 108 & 438 (29) & 635 (53) & $8.70(0.66)$ & $1.1(0.1)$ & $7.9(0.7)$ & $3.31(0.11)$ & $67.0(4.6)$ & $114.6(9.7)$ \\
\hline 66 & 534 (66) & 855 (33) & $9.36(1.2)$ & $1.1(0.0)$ & $8.7(0.5)$ & $7.04(0.48)$ & $61.3(7.6)$ & $94.1(4.9)$ \\
\hline 37 & 642 (123) & $697(77)$ & $12.3(0.01)$ & $1.1(0.2)$ & $10.3(1.1)$ & $5.66(1.1)$ & $44.2(8.5)$ & $44.9(5.0)$ \\
\hline 20 & $750(79)$ & $591(76)$ & $5.50(0.03)$ & $0.9(0.1)$ & $6.4(0.6)$ & $7.12(0.52)$ & $60.2(6.4)$ & $43.0(5.8)$ \\
\hline 14 & 753 (342) & $980(79)$ & $9.50(0.95)$ & $1.1(0.1)$ & $8.6(0.6)$ & $5.48(0.34)$ & $27.4(1.5)$ & $33.0(3.0)$ \\
\hline 10 & $832(28)$ & 3240 (613) & $10.6(0.65)$ & $1.3(0.1)$ & $8.5(0.8)$ & $3.73(0.12)$ & $13.8(0.9)$ & $46.5(8.9)$ \\
\hline 6 & $1330(492)$ & $1220(215)$ & $10.3(0.86)$ & $1.2(0.1)$ & $8.5(0.1)$ & $4.31(0.02)$ & $11.5(3.0)$ & $8.7(1.6)$ \\
\hline \multicolumn{9}{|c|}{ Ammonium $\left(\mathrm{NH}_{4}^{+}\right)$} \\
\hline 150 & $\mathrm{~nm}$ & $669(30)$ & $\mathrm{nm}$ & $\mathrm{nm}$ & $\mathrm{nm}$ & $\mathrm{nm}$ & $\mathrm{nm}$ & $89.8(4.4)$ \\
\hline 108 & $448(44)$ & $643(17)$ & $8.89(0.56)$ & $1.2(0.1)$ & $7.8(0.8)$ & $2.72(0.01)$ & $75.9(7.5)$ & $105.2(3.4)$ \\
\hline 66 & 873 (183) & $668(21)$ & $10.5(0.66)$ & $1.2(0.2)$ & $8.7(1.2)$ & $6.45(0.26)$ & $111.3(23.5)$ & $103.6(3.6)$ \\
\hline 37 & $685(46)$ & $524(32)$ & $10.6(0.88)$ & $1.3(0.1)$ & $8.4(0.5)$ & $7.76(0.16)$ & $70.9(4.9)$ & $42.2(2.7)$ \\
\hline 20 & $1430(234)$ & 954 (112) & $10.0(1.0)$ & $1.2(0.2)$ & $8.3(0.5)$ & $8.51(0.00)$ & $85.8(14.2)$ & $86.8(7.0)$ \\
\hline 14 & $1300(413)$ & $908(62)$ & $9.50(1.3)$ & $1.0(0.1)$ & $9.8(0.2)$ & $4.61(0.07)$ & $56.5(12.8)$ & $41.2(2.9)$ \\
\hline 10 & 2730 (158) & $1090(233)$ & $9.17(1.0)$ & $1.2(0.2)$ & $7.7(0.7)$ & $3.54(0.24)$ & $52.7(4.2)$ & $16.2(3.5)$ \\
\hline 6 & $\mathrm{~nm}$ & $1920(141)$ & $7.90(1.6)$ & $1.1(0.2)$ & $6.9(0.4)$ & 5.44 (N/A) & $\mathrm{nm}$ & $10.7(0.9)$ \\
\hline
\end{tabular}




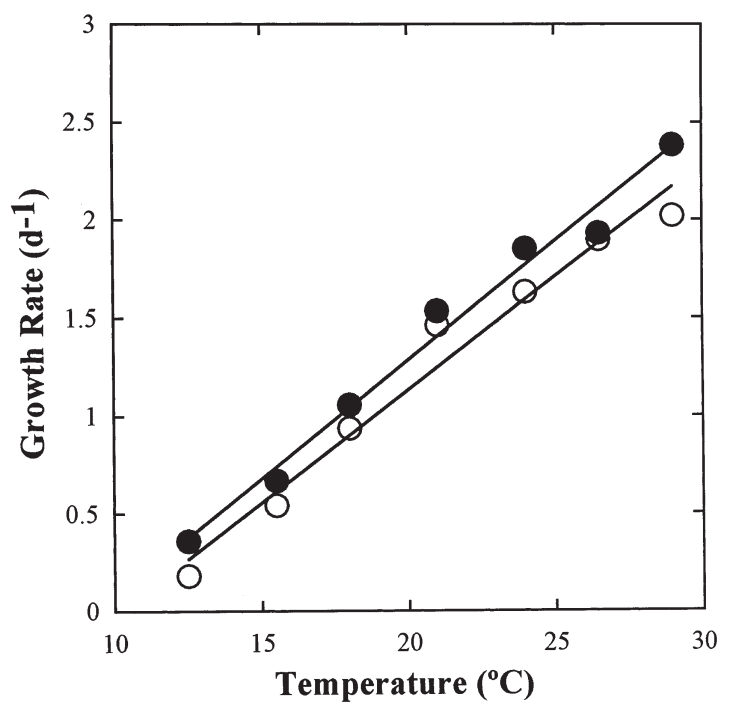

Fig. 4. Thalassiosira weissflogii. Effect of temperature on specific growth rates $\left(\mathrm{d}^{-1}\right)$ of diatoms grown on Fe-sufficient $(\mathrm{pFe}$ 18) Aquil medium amended with either $100 \mu \mathrm{M} \mathrm{NO}_{3}{ }^{-}(\mathrm{O})$ or $\mathrm{NH}_{4}{ }^{+}(-)$at saturating irradiance. SEs around mean were smaller than symbols for all data $(n=4)$

\section{Temperature experiment}

Specific growth rates of Thalassiosira weissflogii increased linearly with increasing temperature $\left(\mathrm{r}^{2}>\right.$ 0.97) (Fig. 4). Although cells in the $\mathrm{NH}_{4}{ }^{+}$-amended medium grew consistently faster over the entire temperature range, they were only significantly faster than the $\mathrm{NO}_{3}{ }^{-}$-grown cells at 13,24 and $29^{\circ} \mathrm{C}$ (ANOVA, $\mathrm{p}<0.05$; Tukey test, $\mathrm{p}<0.05$ ). The $Q_{10}$ values for growth rate were 3.1 and 2.8 for $\mathrm{NO}_{3}{ }^{-}$- and $\mathrm{NH}_{4}{ }^{+}$-grown cultures, respectively. Attempts to cul-

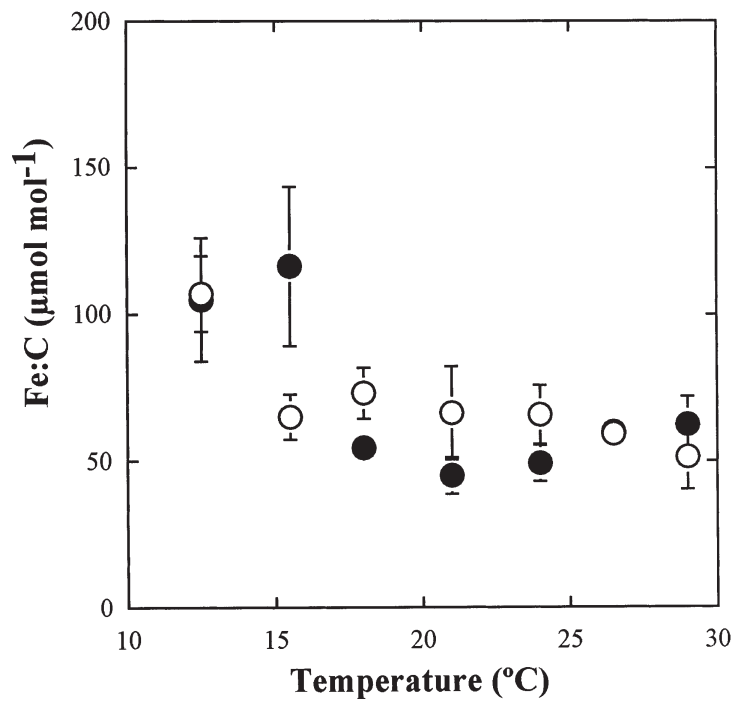

Fig. 5. Thalassiosira weissflogii. Mean Fe:C ratios ( $\mu$ mol Fe $\mathrm{mol}^{-1} \mathrm{C}$ ) of diatoms grown on $100 \mu \mathrm{M} \mathrm{NO}_{3}{ }^{-}$(O) or $\mathrm{NH}_{4}{ }^{+}(\mathbf{O})$ over range of temperatures at saturating irradiance. Error bars: SEs around mean (smaller than symbol when not visible) $(\mathrm{n}=4)$

ture this species at temperatures below $7.5^{\circ} \mathrm{C}$ were unsuccessful.

Iron:carbon of Thalassiosira weissflogii did not vary with temperature or $\mathrm{N}$ substrate between 18 and $29^{\circ} \mathrm{C}$ (Fig. 5). Ammonium-grown cells at $16^{\circ} \mathrm{C}$ exhibited Fe:C ratios that were both significantly higher than at other temperatures, and higher than $\mathrm{NO}_{3}{ }^{-}$-grown cells grown at $16^{\circ} \mathrm{C}$. Ammonium-grown cells at $13^{\circ} \mathrm{C}$ also had Fe:C ratios that were significantly higher than $\mathrm{NH}_{4}{ }^{+}$-grown cells at higher temperatures. Nitrategrown cells at $13^{\circ} \mathrm{C}$ had higher Fe: $\mathrm{C}$ ratios than $\mathrm{NO}_{3}{ }^{-}-$

Table 2. Thalassiosira weissflogii. Physiological and biochemical composition parameters of diatoms grown on either $100 \mathrm{\mu M} \mathrm{NO}_{3}{ }^{-}$or $\mathrm{NH}_{4}{ }^{+}$over range of temperatures. Values within parentheses: $\mathrm{SE}(\mathrm{n}=3$ or 4$)$

\begin{tabular}{|c|c|c|c|c|c|c|}
\hline $\begin{array}{l}\text { Temperature } \\
\left({ }^{\circ} \mathrm{C}\right)\end{array}$ & $\begin{array}{c}\mathrm{Fe} \\
\left(\mathrm{amol} \mathrm{cell}^{-1}\right)\end{array}$ & $\begin{array}{c}\mathrm{C} \\
\left(\mathrm{pmol} \mathrm{cell}^{-1}\right)\end{array}$ & $\begin{array}{c}\mathrm{N} \\
\left(\text { pmol cell }^{-1}\right)\end{array}$ & $\begin{array}{c}\mathrm{C}: \mathrm{N} \\
\left(\mathrm{mol} \mathrm{mol}^{-1}\right)\end{array}$ & $\begin{array}{c}\text { Chl a } \\
\left(\text { pg cell }^{-1}\right)\end{array}$ & $\begin{array}{c}\text { Fe uptake rate } \\
\left(\mu \mathrm{mol} \mathrm{Fe} \mathrm{mol}^{-1} \mathrm{C} \mathrm{d}^{-1}\right)\end{array}$ \\
\hline \multicolumn{7}{|l|}{ Nitrate $\left(\mathrm{NO}_{3}{ }^{-}\right)$} \\
\hline 29 & 447 (88) & $8.34(0.65)$ & $1.1(0.1)$ & $7.5(0.3)$ & $4.53(0.19)$ & $108.4(22.5)$ \\
\hline 27 & $406(38)$ & $6.86(0.50)$ & $0.7(0.1)$ & $9.9(0.6)$ & $4.09(0.10)$ & $112.7(3.5)$ \\
\hline 24 & $461(79)$ & $6.81(0.41)$ & $0.6(0.1)$ & $10.5(0.3)$ & $3.54(0.17)$ & $110.5(16.4)$ \\
\hline 21 & $322(55)$ & $4.81(0.34)$ & $0.6(0.0)$ & $8.5(0.8)$ & $3.48(0.04)$ & $97.3(23.3)$ \\
\hline 18 & $354(4)$ & $5.27(0.12)$ & $0.5(0.1)$ & $10.1(0.6)$ & $1.62(0.20)$ & $62.9(8.2)$ \\
\hline 16 & 547 (95) & $8.23(0.33)$ & $0.7(0.0)$ & $11.4(0.4)$ & $2.01(0.21)$ & $36.0(4.2)$ \\
\hline 13 & $596(34)$ & $4.53(0.66)$ & $0.4(0.1)$ & $10.7(0.7)$ & $0.82(0.02)$ & $24.0(2.4)$ \\
\hline \multicolumn{7}{|c|}{ Ammonium $\left(\mathrm{NH}_{4}{ }^{+}\right)$} \\
\hline 29 & $358(22)$ & $5.77(0.27)$ & $0.6(0.1)$ & $9.0(0.3)$ & $3.63(0.39)$ & $148.0(23.1)$ \\
\hline 27 & $278(11)$ & $4.64(0.05)$ & $0.5(0.1)$ & $9.4(0.9)$ & $2.81(0.15)$ & $115.8(7.3)$ \\
\hline 24 & $310(32)$ & $6.35(0.52)$ & $0.8(0.1)$ & $8.8(0.9)$ & $2.92(0.23)$ & $90.4(11.6)$ \\
\hline 21 & 227 (15) & $5.09(0.40)$ & $0.5(0.0)$ & $10.2(0.6)$ & $2.35(0.10)$ & $68.3(9.5)$ \\
\hline 18 & $286(50)$ & $4.61(0.34)$ & $0.5(0.0)$ & $8.6(0.1)$ & $2.22(0.08)$ & $65.6(6.0)$ \\
\hline 16 & 735 (168) & $6.39(0.18)$ & $0.7(0.0)$ & $9.1(0.4)$ & $1.81(0.03)$ & $76.8(18.3)$ \\
\hline 13 & $837(51)$ & $7.93(1.40)$ & $0.8(0.1)$ & $10.0(0.9)$ & $1.44(0.09)$ & $38.0(7.6)$ \\
\hline
\end{tabular}




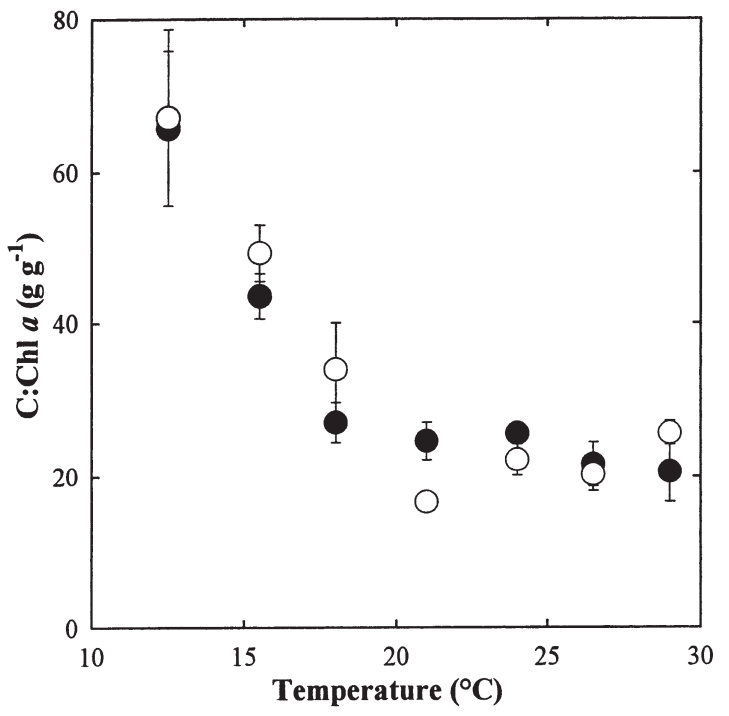

Fig. 6. Thalassiosira weissflogii. Mean C:chlorophyll a ratios $\left(\mathrm{g} \mathrm{g}^{-1}\right)$ of diatoms grown on $100 \mu \mathrm{M} \mathrm{NO}_{3}{ }^{-}$(O) or $\mathrm{NH}_{4}{ }^{+}$ ( ) over range of temperatures at saturating irradiance. Error bars: SE around mean (smaller than the symbol when not visible) $(n=4)$

grown cells at higher temperatures (ANOVA, $\mathrm{p}<0.05$, Tukey test, $\mathrm{p}<0.05)$. Increases in $\mathrm{Fe}$ : $\mathrm{C}$ ratios of $\mathrm{NH}_{4}{ }^{+}$grown cells were a consequence of higher intracellular Fe concentrations (Table 2). Neither temperature nor $\mathrm{N}$ substrate affected $\mathrm{C}$ and $\mathrm{N}$ quotas, or C:N ratio of $T$. weissflogii (2-way ANOVA, p > 0.05; Tukey Test, $\mathrm{p}>$ 0.05) (Table 2). Carbon quotas were highly variable, ranging from 4.53 to $8.34 \mathrm{pmol} \mathrm{cell}^{-1}$. Similarly, N quotas varied from 0.4 to $1.1 \mathrm{pmol} \mathrm{cell}^{-1}$. Variability in $\mathrm{C}$ was often matched by $\mathrm{N}$, resulting in more stable C:N ratios, ranging from 7.5 to $11.4 \mathrm{~mol} \mathrm{C} \mathrm{mol}^{-1} \mathrm{~N}$. Cell volumes $\left(\mu \mathrm{m}^{3}\right)$ were not affected by temperature, but were significantly greater (ANOVA, $\mathrm{p}<0.05$ ) for $\mathrm{NH}_{4}{ }^{+}$grown cells (mean $\pm \mathrm{SE}: \mathrm{NO}_{3}{ }^{-}=1277 \pm 136 ; \mathrm{NH}_{4}{ }^{+}=$ $1700 \pm 153[\mathrm{n}=7])$.

Chl a concentrations (pg cell ${ }^{-1}$ ) decreased at low temperature (Table 2). Chl a was not affected by $\mathrm{N}$ substrate except at 21 and $27^{\circ} \mathrm{C}$, where $\mathrm{NH}_{4}{ }^{+}$-grown cells had significantly higher concentrations (ANOVA, $\mathrm{p}<0.05$, Tukey test, $\mathrm{p}<0.05$ ). Carbon:chl a ratios were relatively invariant between 21 and $29^{\circ} \mathrm{C}$. The decrease in chl a for both $\mathrm{NO}_{3}{ }^{-}$-and $\mathrm{NH}_{4}{ }^{+}$-grown cells below $21^{\circ} \mathrm{C}$ resulted in a linear increase in C:chl a (Fig. 6). When normalized to C, chl a content did not differ significantly between cells grown on $\mathrm{NO}_{3}{ }^{-}$- and $\mathrm{NH}_{4}{ }^{+}$-amended media (ANOVA, $\mathrm{p}>0.05$ ). Inexplicably, C:chl a was ca 2-fold lower in the temperature than in the light experiment at similar irradiance $\left(>100 \mu \mathrm{mol}\right.$ photons $\left.\mathrm{m}^{-2} \mathrm{~s}^{-1}\right)$ and temperature $\left(20^{\circ} \mathrm{C}\right)$.

\section{DISCUSSION}

\section{Effects of light on Fe content and steady-state Fe uptake}

The dependence on Fe for photosynthetic energy conversion stems from its catalytic role in cytochromes and $\mathrm{Fe}-\mathrm{S}$ proteins of the electron-transport chain. Under nutritionally replete conditions, each functional electron-transport chain contains a minimum of $23 \mathrm{Fe}$ atoms, the largest metabolic Fe pool in photoautotrophs. Acclimation to decreasing irradiance results in increased production of light-harvesting pigments (Richardson et al. 1983, Falkowski \& La Roche 1991) and changes in abundance and stoichiometry of the Fe-containing constituents of the photosynthetic apparatus. Theoretical (Raven 1990) and empirical (Kudo \& Harrison 1997, Sunda \& Huntsman 1997) data show that at low light such changes increase the Fe requirements for phytoplankton growth.

Our results are in general agreement with these previous studies, as the Fe quotas of Thalassiosira weissflogii increased significantly as irradiance decreased. Moreover, our results provide the first evidence of a light-induced increase in $\mathrm{Fe}$ quotas for a marine diatom. Although the elevated Fe content of low-light cells is consistent with expectations, quota measurements by themselves provide no information regarding the functional role of this additional Fe. Slower rates of light-limited growth could facilitate Fe accumulation if transport rates are not concomitantly reduced. The unknown quantities are the degree of coupling that exists between transport and growth, and the allocation of cellular Fe into metabolic and non-metabolic pools. In the presence of the high concentrations of Fe in our medium, Fe quotas could increase and the accumulated Fe would not necessarily be biochemically active.

Calculated steady-state rates show that, indeed, Fe accumulation results because growth rates declined more rapidly with light than Fe transport rates (Fig. 7). This observation is consistent with physiological regulation by Thalassiosira weissflogii to meet the increased Fe demand for growth at low light. The results of the temperature experiment provide additional support for this interpretation. They show that over a similar decline in relative growth rates Fe quotas were largely invariant, although they increased somewhat at the lowest temperature tested. Thus, transport of Fe is regulated in a fundamentally different fashion when either light or temperature limits growth. Iron transport is tightly coupled to growth requirements under steady-state conditions and increases the accumulation of Fe at low light, presumably because of the cells greater nutritional requirement. 


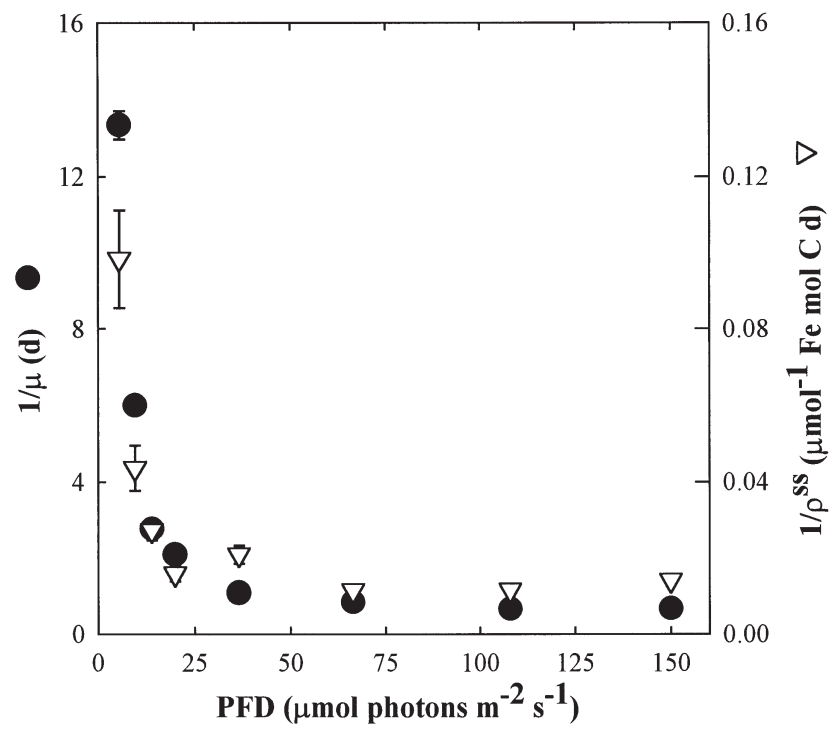

Fig. 7. Thalassiosira weissflogii. Reciprocal mean growth $(1 / \mu ; \bullet)$ and steady-state Fe uptake $\left(1 / \rho^{\mathrm{ss}} ; \nabla\right)$ rates over range of irradiances at $20^{\circ} \mathrm{C}$. Values are means of all treatments $\left(\mathrm{NO}_{3}{ }^{-}\right.$- and $\mathrm{NH}_{4}^{-}$-grown cells, both trials). Error bars: $\mathrm{SE}$ around the mean (smaller than symbol when not visible) (n $=3$ to 4$)$

Part of the low-light-induced increase in Fe quota can be attributed to an increase in reaction center density. We can calculate the amount of Fe associated with photoacclimation in Thalassiosira weissflogii using the data of Dubinsky et al. (1986). According to their results, an increase in cellular chl a was accompanied by a 2-fold increase in PSI abundance as growth irradiance was reduced from 150 to $30 \mu \mathrm{mol}$ photons $\mathrm{m}^{-2} \mathrm{~s}^{-1}$. The cellular abundance of PSII was relatively unaffected over this irradiance range. Assuming an Fe:PSI stoichiometry of $12: 1$ and an Fe:PSII stoichiometry of 3:1 (Raven 1990), these biochemical changes should result in a 1.3-fold increase in Fe requirements for photosynthesis. Remarkably, over a similar irradiance range (150 to $37 \mu \mathrm{mol}$ photons $\mathrm{m}^{-2} \mathrm{~s}^{-1}$ ), our measured increase in Fe quotas is nearly identical to this prediction. Accumulation of Fe under low light can be accounted for, in part, by its requirement in the PSII and PSI complexes. The calculation also shows that more than $20 \%$ of the cellular Fe quota of T. weissflogii is incorporated into these 2 complexes alone. Conspicuously absent from this calculation are data for other Fe-rich components of the photosynthetic electrontransport chain, particularly the cytochrome $b_{6}$ f complex. In the diatom Phaeodactylum tricornutum, for example, this complex accounts for over $60 \%$ of photosynthetic Fe under saturating light and Fe-replete conditions (Greene et al. 1991). Because phytoplankton species have unique strategies for photoacclimation
(Falkowski \& La Roche 1991), we cannot reliably infer photosynthetic Fe requirements of $T$. weissflogii using data from other organisms. Future work should focus on quantifying the major photosynthetic components of $T$. weissflogii and how these change over an irradiance range.

The preceding calculation cannot be made for the lower irradiances, where Fe accumulation was the most substantial: below $37 \mu \mathrm{mol}$ photons $\mathrm{m}^{-2} \mathrm{~s}^{-1}$, Fe quota was as much as 4 times that of the high-light cells. The increase in quota was not associated with an increase in chl a production. The ratio of $\mathrm{C}$ :chl $a$ increased under low light, while Fe:C ratios concomitantly increased. Changes in these ratios were not due to variation in $\mathrm{C}$ quotas, which remained constant over the irradiance range. Rather, Fe quotas increased whereas chl a concentrations decreased under low light. Similar reductions in chl a have been reported previously for marine diatoms (Falkowski \& Owens 1980, Thompson et al. 1989). The low-light chlorosis phenomenon appears restricted to exceptionally low irradiances $\left(<15 \mu \mathrm{mol}\right.$ photons $\left.\mathrm{m}^{-2} \mathrm{~s}^{-1}\right)$. While Fe is known to function in at least 2 catalytic steps in the chlorophyll biosynthesis pathway (Chereskin \& Castelfranco 1982, Spiller et al. 1982), the pool size and turnover rate of these Fe catalysts are not known, although we surmise that they are small and fast. Based on our findings, chlorophyll synthesis, per se, does not increase Fe demand. In Thalassiosira weissflogii, chlorophyll degradation and turnover is slow (Goericke \& Welschmeyer 1992), and decreases in pigment concentration are primarily the result of dilution through cell division and passive degradation. The increase in $\mathrm{Fe}$ : $\mathrm{C}$ is thus more aptly attributable to some other physiological response to low light, but Fe storage in a non-metabolic pool is also possible. Since phytoplankton have considerable variation in their maximum and minimum Fe quotas (Sunda \& Huntsman 1995, Maldonado \& Price 1996) and Fe concentrations in our media were high, this latter suggestion warrants further investigation.

Iron-replete conditions were maintained in the media to minimize the biological effects of having different rates of photoreduction of Fe(III) complexes at each growth irradiance. Because the estimated Fe' concentration (41 nM) computed for Aquil medium in the dark was in excess of concentrations that saturate the growth rate of Thalassiosira weissflogii in the light (Sunda \& Huntsman 1995), any increase in Fe due to photochemical reactions should have had no affect on the Fe status of the cultures. Throughout the entire irradiance range that was employed, Fe was thus saturating for growth. Variation in $\mathrm{Fe}^{\prime}$ in the temperature experiment is even less of a concern as the irradiance was kept constant, reaction quantum yields of Fe(III)- 
EDTA are nearly invariant with temperature (Kari et al. 1995), and changes in the rate of thermal dissolution are negligible over the temperature range tested (Morel \& Hering 1993).

\section{Effects of $\mathrm{N}$ substrate on Fe content}

Increased Fe requirement for $\mathrm{NO}_{3}{ }^{-}$use may arise because the $\mathrm{NO}_{3}{ }^{-}$-assimilatory enzymes nitrate and nitrite reductase are Fe-containing redox enzymes (Wray \& Fido 1990). Additional reductant, in the form of ferredoxin, derived from Fe-dependent photosynthetic redox reactions is also necessary for $\mathrm{NO}_{3}{ }^{-}$reduction. Therefore, phytoplankton Fe requirements should reflect the $\mathrm{N}$ source used for growth. In the Fe-deficient waters of the equatorial Pacific, Fe additions not only enhance net phytoplankton growth and biomass, but also $\mathrm{N}$-specific $\mathrm{NO}_{3}{ }^{-}$-uptake rates of the phytoplankton community (Price et al. 1991, 1994). Laboratory experiments demonstrate that growth on $\mathrm{NO}_{3}{ }^{-}$imparts both higher Fe:C ratios and higher steady-state Fe uptake rates when $\mathrm{Fe}$ is limiting (Maldonado \& Price 1996). The lack of difference between nitrate- and ammonium-grown cells reported here leads us to believe that excess accumulation of Fe in a non-metabolic pool may have masked the $\mathrm{N}$ effect. Maldonado \& Price found that the effect of $\mathrm{N}$ substrate on Fe quotas was only evident under Fe-limiting conditions. Indeed, the increased Fe demand for nitrate assimilation is approximately 3-fold lower than the estimated increase in Fe demand for acclimation to low light (ca 40 and $120 \mathrm{amol} \mathrm{cell}^{-1}$ more Fe, respectively). As in this study, Maldonado \& Price found no N substrate effect on the Fe quotas of Fe-replete T. weissflogii cells.

\section{Effects of temperature on Fe content}

In many ways, temperature and light acclimation are analogous processes; both are regulated by the oxidation state of the plastoquinone pool (i.e., the excitation pressure on PSII) (Maxwell et al. 1994, 1995a,b, Savitch et al. 1996) and both may result in changes to cellular chlorophyll concentrations (Geider 1987, Falkowski \& LaRoche 1991). Low temperature constrains the rate of carbon fixation by decreasing enzyme activities, causing PSII excitation pressure to increase as it does under high light. From a photo-physiological point of view, low temperature- and high-light acclimated phytoplankton are expected to have similarities. Indeed, acclimation to low temperature commonly involves increases in abundance of Calvin cycle enzymes (Li \& Morris 1982, Mortain-Bertrand et al. 1988, Savitch et al. 1996) and alterations to the photosyn- thetic apparatus (Davison 1991, Maxwell et al. 1994, 1995a,b). Decreases in PSII chlorophyll protein complexes (LHCII) and $\mathrm{chl} a / b$ binding protein mRNA are observed at low temperature in some phototrophs (Maxwell et al. 1995a,b). In contrast, the effects of temperature on the photosynthetic electron-transport chain are less clear. Photosystem abundance has been reported to decrease slightly or remain constant with decreasing temperature (Davison 1991). Since the reaction centers are dependent on $\mathrm{Fe}$, and enzymes of carbon fixation are not, the Fe requirements of phytoplankton could be affected by the strategy of low temperature acclimation. Considering all possible combinations of biochemical changes that could occur, Fe quotas are likely to remain relatively unchanged or to decrease as temperature declines. For Thalassiosira weissflogii, the results suggest that $\mathrm{Fe}$ C ratios are largely invariant across the range of temperatures we employed. Thus, if Fe:C ratios mirror photosynthetic Fe requirements, only small changes in the densities of the photosystem components are likely to have occurred. Decreases in cellular chl a are consistent with previous observations (Geider 1987). As argued above for the light experiments, such changes should have a negligable affect on the Fe requirements of $T$. weissflogii.

\section{Oceanographic implications}

The results of our study confirm additional cellular Fe content for Thalassiosira weissflogii grown at low light. They show that Fe uptake rates are maintained in excess of growth rates under low light, so that Fe accumulates and Fe:C ratios increase. Part of the increase can be attributed to changes in the reaction center components, although, in the presence of high Fe concentrations, Fe quotas may exceed those necessary for photosynthetic metabolism. The implications of our findings are that photoacclimation of phytoplankton could be affected by the availability of Fe and that both the onset and extent of Fe limitation in natural systems could be modulated by light.

The growth of coastal phytoplankton assemblages can be limited by the availability of Fe. Hutchins \& Bruland (1998) demonstrated that portions of the California coastal upwelling regime are similar to oceanic high-nitrate, low-chlorophyll a (HNLC) regions. Adding Fe to samples collected from these coastal regions promotes blooms of large diatoms. The natural supply of Fe appears to come from upwelled water enriched in coastalshelf sediments (Johnson et al. 1999). Interpretation of the photoacclimatory response of the Fe-limited diatom community to this upwelled Fe-rich water is complicated for several reasons. Resuspension of coastal sedi- 
ments, in addition to supplying $\mathrm{Fe}$, also leads to markedly lower light transmission (Johnson et al. 1999). Reduced light transmission would act to both decrease rates of photoreductive dissolution of Fe colloids and organic Fe complexes and increase Fe demands for photoacclimation. Photosynthetic Fe demands will also be influenced by the extent to which coastal diatoms are able to take up and store Fe in excess of metabolic requirements (Sunda et al. 1991, Sunda \& Huntsman 1995, Maldonado \& Price 1996).

Photosynthetic parameters of phytoplankton in oceanic HNLC regions provide some information on the Fe costs for photoacclimation. Cullen et al. (1992) concluded that phytoplankton assemblages from the equatorial Pacific were well adapted to the ambient light regime, although Fe limited their growth (Kolber et al. 1994). They showed that light-saturated rates of photosynthesis $\left(P_{m}{ }^{\mathrm{chl}}\right)$ and the saturation parameter $I_{k}\left(P_{m}{ }^{\text {chl }} / \alpha\right)$ decreased with depth, as expected for a light-acclimated community. Since upwelling provides the majority of $\mathrm{Fe}$ in these waters (Coale et al. 1996), deep-living populations may have access to higher concentrations to support their greater demands. High incident irradiance and a shallow mixed-layer depth should certainly act to reduce the degree to which phytoplankton need to acclimate to low ambient light and hence require Fe. In the Southern Ocean, however, the situation is more extreme. Light limitation is pronounced because of extensive wind mixing in combination with dense clouds and seasonal ice cover (Mitchell et al. 1991, Nelson \& Smith 1991). The requirement for $\mathrm{Fe}$ for photoacclimation should thus be greatly enhanced. Observations of Sakshaug \& Holm-Hansen (1986) are consistent with this in that they show that $P_{m}{ }^{\text {chl }}$ is invariant with depth in stratified surface waters. The Southern Ocean phytoplankton community is not always well adapted to the prevailing light conditions, possibly because of insufficient Fe. In subarctic Pacific waters, metabolic rates, growth, and photosynthetic parameters of phytoplankton are enhanced in the winter by increasing irradiance or Fe (Maldonado et al. 1999). Moreover, the greatest physiological enhancements occur when Fe and light are provided concomitantly. Thus, in this HNLC region, light and Fe can be co-limiting resources.

Phytoplankton acclimate to low light using fundamentally different strategies that may even vary among closely related species and ecotypes (Sakshaug et al. 1987, Falkowski \& La Roche 1991). The extent to which irradiance affects phytoplankton Fe requirements should thus be influenced by the strategies they employ, and possibly by differences in their Fe requirements for growth and capacities for luxury consumption and storage of Fe (Sunda et al. 1991, Sunda \& Huntsman 1995, Maldonado \& Price 1996). Future experiments should ex- amine taxa isolated from environments like the Southern Ocean, where survival requires adaptation to both perennially low irradiances and sporadic Fe input.

Acknowledgements. We thank Drs M. Maldonado and A. Milligan for providing helpful comments. Funding for this work was obtained through grants from the Natural Sciences and Engineering Council of Canada and the McGill University Faculty of Graduate Studies and Research.

\section{LITERATURE CITED}

Behrenfeld MJ, Bale AJ, Kolber ZS, Aiken J, Falkowski PG (1996) Confirmation of iron limitation of phytoplankton photosynthesis in the equatorial Pacific Ocean. Nature 383:508-511

Booth BC (1987) The use of autofluorescence for analyzing oceanic phytoplankton communities. Bot Mar 30:101-108

Brand LE, Guillard RRL, Murphy LS (1981) A method for the rapid and precise determination of acclimated phytoplankton reproductive rate. J Plankton Res 3:193-201

Chereskin B, Castelfranco P (1982) Effects of iron and oxygen on chlorophyll biosynthesis. II. Observations on the biosynthetic pathway in isolated etiochloroplasts. Plant Physiol 69:112-116

Coale KH, Fitzwater SE, Gordon RM, Johnson KS, Barber RT (1996) Control of community growth and export production by upwelled iron in the equatorial Pacific Ocean. Nature 379:621-624

Cullen JJ, Lewis MR, Davis CO, Barber RT (1992) Photosynthetic characteristics and growth rates indicate grazing is the proximate control of primary production in the equatorial Pacific. J Geophys Res 97:639-654

Davison IR (1991) Environmental effects on algal photosynthesis: temperature. J Phycol 27:2-8

Dubinsky Z, Falkowski PG, Wyman K (1986) Light harvesting and utilization by phytoplankton. Plant Cell Physiol 27: 1335-1349

Falkowski PG, La Roche J (1991) Acclimation to spectral irradiance in algae. J Phycol 27:8-14

Falkowski PG, Owens TG (1980) Light-shade adaptation. Two strategies in marine phytoplankton. Plant Physiol 66: 592-595

Falkowski PG, Owens TG, Ley AC, Mauzerall DC (1981) Effects of growth irradiance on the ratios of reaction centers in two species of marine phytoplankton. Plant Physiol 68:969-973

Friedman AL, Alberte RS (1986) Biogenesis and light regulation of the major light harvesting chlorophyll-protein of diatoms. Plant Physiol 80:43-51

Geider RJ (1987) Light and temperature dependence of the carbon to chlorophyll a ratio in microalgae and cyanobacteria: implications for physiology and growth of phytoplankton. New Phytol 106:1-34

Geider RJ, La Roche J, Greene RM, Olaizola M (1993) Response of the photosynthetic apparatus of Phaeodactylum tricornutum (Bacillariophyceae) to nitrate, phosphate, or iron starvation. J Phycol 29:755-766

Goericke R, Welschmeyer NA (1992) Pigment turnover in the marine diatom Thalassiosira weissflogii. I. The ${ }^{14} \mathrm{CO}_{2}$-labeling kinetics of chlorophyll a. J Phycol 28: 498-507

Greene RM, Geider RJ, Falkowski PG (1991) Effect of iron on photosynthesis in a marine diatom. Limnol Oceanogr 36: 1772-1782 
Greene RM, Geider RJ, Kolber Z, Falkowski PG (1992) Ironinduced changes in light harvesting and photochemical energy conversion processes in eukaryotic marine algae. Plant Physiol 100:565-575

Hudson RJ, Morel FMM (1989) Distinguishing between extraand intracellular iron uptake in marine phytoplankton. Limnol Oceanogr 34:1113-1120

Hutchins DA, Bruland KW (1998) Iron-limited diatom growth and Si:N uptake ratios in a coastal upwelling regime. Nature 393:561-564

Johnson KS, Chavez FP, Friederich GE (1999) Continentalshelf sediment as a primary source of iron for coastal phytoplankton. Nature 398:697-700.

Kari FG, Hilger S, Canonica S (1995) Determination of the reaction quantum yield for the photochemical degradation of Fe(III)-EDTA: implications for the environmental fate of EDTA in surface waters. Environ Sci Technol 29: 1008-1017

Kolber Z, Barber RT, Coale KH, Fitzwater SE, Greene RM, Johnson KS, Lindley S, Falkowski PG (1994) Iron limitation of phytoplankton photosynthesis in the equatorial Pacific Ocean. Nature 371:145-149

Kudo I, Harrison PJ (1997) Effect of iron nutrition on the marine cyanobacterium Synechococcus grown on different N sources and irradiances. J Phycol 33:232-240

Li WKW, Morris I (1982) Temperature adaptation in Phaeodactylum tricornutum Bohlin: photosynthetic rate compensation and capacity. J Exp Mar Biol Ecol 58:135-150

Maldonado MT, Price NM (1996) Influence of N substrate of Fe requirements of marine centric diatoms. Mar Ecol Prog Ser 141:161-172

Maldonado MT, Boyd PW, Harrison PJ, Price NM (1999) Colimitation of phytoplankton growth by light and Fe during winter in the NE subarctic Pacific Ocean. Deep-Sea Res II 46:2475-2485

Maxwell DP, Falk S, Trick CG, Huner NPA (1994) Growth at low temperature mimics high-light acclimation in Chlorella vulgaris. Plant Physiol 105:535-543

Maxwell DP, Falk S, Huner NPA (1995a) Photosystem II excitation pressure and development of resistance to photoinhibition I. LHCII abundance and zeaxanthin content in Chlorella vulgaris. Plant Physiol 107:687-694

Maxwell DP, Laudenbach DE, Huner NPA (1995b) Redox regulation of light-harvesting complex II and cab mRNA abundance in Dunaliella salina. Plant Physiol 109:787-795

Mitchell BG, Brody EA, Holm-Hansen O, McClain C, Bishop J (1991) Light limitation of phytoplankton biomass and macronutrient utilization in the Southern Ocean. Limnol Oceanogr 36:1662-1677

Morel FMM, Hering JG (1993) Principles and applications of aquatic chemisty. Wiley-Interscience, New York

Morel FMM, Reuter JG, Anderson DM, Guillard RRL (1979) Aquil: a chemically defined phytoplankton culture medium for trace metal studies. Limnol Oceanogr 36: $1742-1755$

Mortain-Bertrand A, Descolas-Gros C, Jupin H (1988) Growth, photosynthesis and carbon metabolism in the temperate marine diatom Skeletonema costatum adapted to low temperature and low photon-flux density. Mar Biol 100:135-141

Neale PJ, Melis A (1986) Algal photosynthetic membrane complexes and the photosynthesis-irradiance curve: a comparison of light-adaptation responses in Chlamydomonas reinhardtii (Chlorophyta). J Phycol 22:531-538

Nelson DM, Smith WO (1991) Sverdrup revisited: critical depths, maximum chlorophyll levels, and the control of Southern Ocean productivity by the irradiance-mixing regime. Limnol Oceanogr 36:1650-1661

Parsons TR, Maita Y, Lalli CM (1984) A manual of chemical and biological methods for seawater analysis. Pergamon Press, Oxford

Price NM, Harrison GI, Hering JG, Hudson RJ, Nirel PMV, Palenik B, Morel FMM (1988/1989) Preparation and chemistry of the artificial algal culture medium Aquil. Biol Oceanogr 6:443-461

Price NM, Anderson LF, Morel FMM (1991) Iron and nitrogen nutrition of equatorial Pacific plankton. Deep-Sea Res 38: 1361-1378

Price NM, Ahner BA, Morel FMM (1994) The equatorial Pacific Ocean: Grazer-controlled phytoplankton populations in an iron-limited ecosystem. Limnol Oceanogr 39: $520-534$

Platt T, Gallegos CL (1980) Modeling primary production. In: Falkowski PG (ed) Primary production in the sea. Environmental Science Research, Vol 19. Plenum Press, New York, p 339-362

Raven JA (1990) Predictions of Mn and Fe use efficiencies of phototrophic growth as a function of light availability for growth and of C assimilation pathway. New Phytol 116: $1-18$

Richardson K, Beardall J, Raven JA (1983) Adaptation of unicellular algae to irradiance: an analysis of strategies. New Phytol 93:157-191

Ringbom A (1963) Complexation in analytical chemistry. Interscience, New York

Sakshaug E, Holm-Hansen O (1986) Photoadaptation in Antarctic phytoplankton: variations in growth rate, chemical composition and P versus I curves. J Plankton Res 8: $459-473$

Sakshaug E, Demers S, Yentsch CM (1987) Thalassiosira oceanica and T. pseudonana: two different photoadaptational responses. Mar Ecol Prog Ser 41:275-282

Savitch LV, Maxwell DP, Huner NPA (1996) Photosystem II excitation pressure and photosynthetic carbon metabolism in Chlorella vulgaris. Plant Physiol 111:127-136

Spiller SC, Castelfranco A, Castelfranco P (1982) Effects of iron and oxygen on chlorophyll biosynthesis. I. In vivo observations of iron and oxygen-deficient plants. Plant Physiol 69:107-111

Sunda WG, Huntsman SA (1995) Iron uptake and growth limitation in oceanic and coastal phytoplankton. Mar Chem 50:189-206

Sunda WG, Huntsman SA (1997) Interrelated influence of iron, light and cell size on marine phytoplankton growth. Nature 390:389-392

Sunda WG, Swift DG, Huntsman SA (1991) Low iron requirement for growth in oceanic phytoplankton. Nature 351: 55-57

Thompson PA, Levasseur ME, Harrison PJ (1989) Light-limited growth on ammonium vs. nitrate: what is the advantage for marine phytoplankton? Limnol Oceanogr 34: 1014-1024

Wells ML, Price NM, Bruland KW (1995) Iron chemistry in seawater and its relationship to phytoplankton: a workshop report. Mar Chem 48:157-182

Westall JC, Zachary JL, Morel FMM (1976) MINEQL: a computer program for the calculation of chemical equilibrium composition of aqueous systems. R. M. Parsons Laboratory for Water Resources and Environmental Engineering, MIT, Department of Civil Engineering, Boston (Tech Note No. 18)

Wray JL, Fido RJ (1990) Nitrate reductase and nitrite reductase. In: Dey PM, Harborne JB (eds) Methods in plant biochemistry. Academic Press, London, p 241-256 